TRABAJOS DE PREHISTORIA

58, n. $^{\circ} 2,2001$, pp. 129 a 142

\title{
NOTICIARIO
}

\section{LA CUEVA DE LOS MOROS DE SAN VITORES (MEDIO CUDEYO). UNA NUEVA ESTACIÓN DE ARTE RUPESTRE PALEOLÍTICO EN CANTABRIA}

\author{
LOS MOROS DE SAN VITORES CAVE (MEDIO CUDEYO). A NEW SITE \\ OF PALEOLITHIC ROCK ART IN CANTABRIA
}

RAMÓN MONTES BARQUÍN $(*)$
EMILIO MUÑOZ FERNANDEZ $(*)$
JOSÉ M. MORLOTE EXPÓSITO $\left(^{*}\right)$

\section{RESUMEN}

Se presenta un nuevo conjunto parietal paleolítico descubierto recientemente en la cueva denominada "Los Moros de San Vitores". Este conjunto se compone de puntos y manchas rojas localizados en el vestíbulo de la cavidad y de un panel con grabados figurativos, cuatro cuadrúpedos y un posible signo, ubicado en el tramo final de la misma. El conjunto de grabados, de gran homogeneidad formal y estilística, es atribuido al Magdaleniense.

\begin{abstract}
We are introducing a new example of paleolithic art, recently discovered in the cave known as 'Los Moros de San Vitores'. This example consists of dots and red patches located in the main entrance of the cave and a panel with four engraved animals and a kind of sign, situated on its last part. All these engravings, with their great stylistic and formal similarities, belong to the Magdalenian period.

Palabras clave: Cantabria. Arte rupestre paleolítico. Cueva de Los Moros de San Vitores. Grabados parietales. Magdaleniense.
\end{abstract}

Key words: Cantabria. Paleolithic rock art. Los Moros de San Vitores cave. Wall engravings. Magdalenian period.

(*) GAEMarqueólogos. José Barros, 1 - local 26. 39600 Muriedas. Cantabria. Correo electrónico: gaem_arqueologos@ inicia.es

El artículo fue remitido en su versión final el 24-IV-2001.

\section{INTRODUCCIÓN}

Durante el año 1996, la Consejería de Cultura y Deportes del Gobierno de Cantabria realizó un inventario exhaustivo de las cavidades con indicios y/o evidencias deArte Rupestre Paleolítico que aún no poseían expediente de incoación abierto para su declaración como Bien de Interés Cultural (B.I.C.).

Este inventario tenía como objetivo, además de completar la relación de elementos del Patrimonio Cultural susceptibles de ser incoados como B.I.C. en Cantabria, el preparar el catálogo de cavidades con vestigios artísticos del Paleolítico al objeto de proceder, conjuntamente con las comunidades de Asturias y País Vasco, a solicitar a la UNESCO la declaración de Patrimonio de la Humanidad para las cuevas con arte rupestre paleolítico de la Región Cantábrica. Con estos objetivos, el equipo de arqueólogos firmante fue contratado con el fin de revisar una serie de cavidades en las que, en alguna ocasión, se hubieran citado indicios de la existencia de manifestaciones artísticas de esta cronología. En ese listado figuraba la gruta conocida como "Los Moros de San Vitores".

En el caso de esta cavidad, el objetivo de la revisión era el de documentar dos puntos o manchas rojas descubiertas y citadas por el Colectivo para la Ampliación de Estudios de Arqueología Prehistórica (Muñoz et alii, 1988) y verificar su posible cronología paleolítica. Durante la revisión, además de confirmar la existencia de estas manchas rojas y establecer una probable cronología paleolítica para las mismas, se detectó la existencia de un pequeño conjunto de grabados. Ante la evidencia de que se 


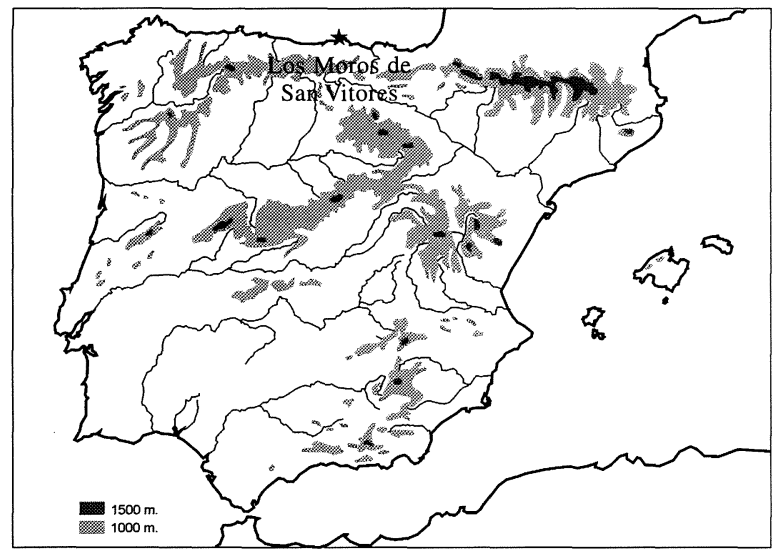

Fig. 1. Localización de la cueva de Los Moros de San Vitores (Medio Cudeyo, Cantabria).

trataba de manifestaciones indudablemente prehistóricas, y de que eran observables varias figuras de cuadrúpedos, se procedió a efectuar un detenido proceso de documentación y validación del conjunto al objeto de incorporar la cavidad al registro de cavidades con arte rupestre paleolítico susceptibles de ser incoadas como B.I.C.

Una vez cumplido este objetivo, y después de que la Consejería de Cultura y Deporte dotase recientemente a la cavidad de una verja para su protección, procedemos ahora a presentar el hallazgo a la comunidad científica.

\section{LOCALIZACIÓN Y DESCRIPCIÓN DE LA CAVIDAD}

La cueva de Los Moros se localiza en el tramo inferior de la cuenca de Miera, en las estribaciones de la ladera sur de Peña Cabarga, encima del núcleo rural de San Vitores. Sus coordenadas U.T.M. son (hoja 1: 5.000 , número VII-26): 437.875, 4.802.480, $\mathrm{z}=190 \mathrm{~m}$. (Fig. 1).

Su ubicación cabe ser calificada como de estratégica ya que desde la cueva se posee un amplio control visual del valle bajo del Miera, y en general de la comarca de Trasmiera, zona que cabe ser definida como muy apta para la subsistencia de los grupos humanos, en especial de los grupos cazadores-recolectores, por sus excelentes y variadas condiciones biogeográficas.

La boca de la cueva se abre en un pequeño farallón calizo localizado a escasos $10 \mathrm{~m}$ por encima del trazado del antiguo ferrocarril minero que discurría por las faldas de Peña Cabarga, el cual permitía el transporte del mineral de hierro por la sierra a finales del siglo XIX e inicios del XX.

Para acceder a la misma se toma un camino que, desde la iglesia de la localidad de San Vitores, accede a las cabañas situadas en la parte alta del pueblo. Desde aquí, hay que continuar el camino a pie, ascendiendo las primeras pendientes de la Peña Cabarga hasta la desmantelada vía férrea. Una vez alcanzada ésta, se toma un sendero ascendente que lleva directamente a la cueva.

La cavidad se abre en calizas aptienses (Cretácico Inferior), que han formado en la zona un abrupto modelado kárstico de tipo lenar, donde además de la cueva objeto de este estudio, se localizan abundantes cavernamientos de pequeño tamaño. Se trata de un sumidero fósil, de unos $65 \mathrm{~m}$ de desarrollo y amplias proporciones, en el que son frecuentes las formaciones litogenéticas en proceso reconstructivo. Su relleno sedimentario está compuesto, principalmente, de arcillas muy ricas en componentes férricos, por lo que toda la cavidad presenta una intensa tonalidad rojiza.

La gruta posee una boca amplia, de unos $14 \mathrm{~m}$. de anchura por $3 \mathrm{~m}$ de altura máxima, y está orientada al S-SE. La misma ha sido parcialmente tapiada con un muro de sillarejo que deja abierto un vano, a manera de puerta, en su parte derecha. Este muro permitía el uso del abrigo de la cavidad como aprisco de ganado menor (cabras) y como refugio de pastores.

El vestíbulo, dividido en dos por otro pequeño murete, presenta $6 \mathrm{~m}$ de profundidad en la parte izquierda, y $9 \mathrm{~m}$ en la derecha. Se trata de un espacio amplio, similar en amplitud a la boca, que va descendiendo progresivamente en altura desde los $3 \mathrm{~m}$ de la zona exterior hasta casi cegarse en el fondo. En este último punto se han formado gran cantidad de espeleotemas que dejan pequeños pasos hacia el interior de la gruta; a través de ellos se accede a una sala de gran tamaño. El paso más transitable está en la derecha del vestíbulo, en un estrechamiento de 1,80 m de anchura y menos de un metro de altura. En la parte izquierda del vestíbulo hay otros tres pasos de menores dimensiones, desde los que se accede a un cono de derrubios con caída hacia el interior.

La única sala del interior es de grandes dimensiones, con un desarrollo total de $39 \mathrm{~m}$ de longitud y una anchura de hasta $25 \mathrm{~m}$ en la zona más próxima al vestíbulo, y unos $10 \mathrm{~m}$ en el fondo. La altura de la sala varía entre los $2 \mathrm{~m}$ del inicio y los 14 $\mathrm{m}$ del fondo, siendo muy frecuentes las formacio-

T. P., 58, n. $^{\circ} 2,2001$ 


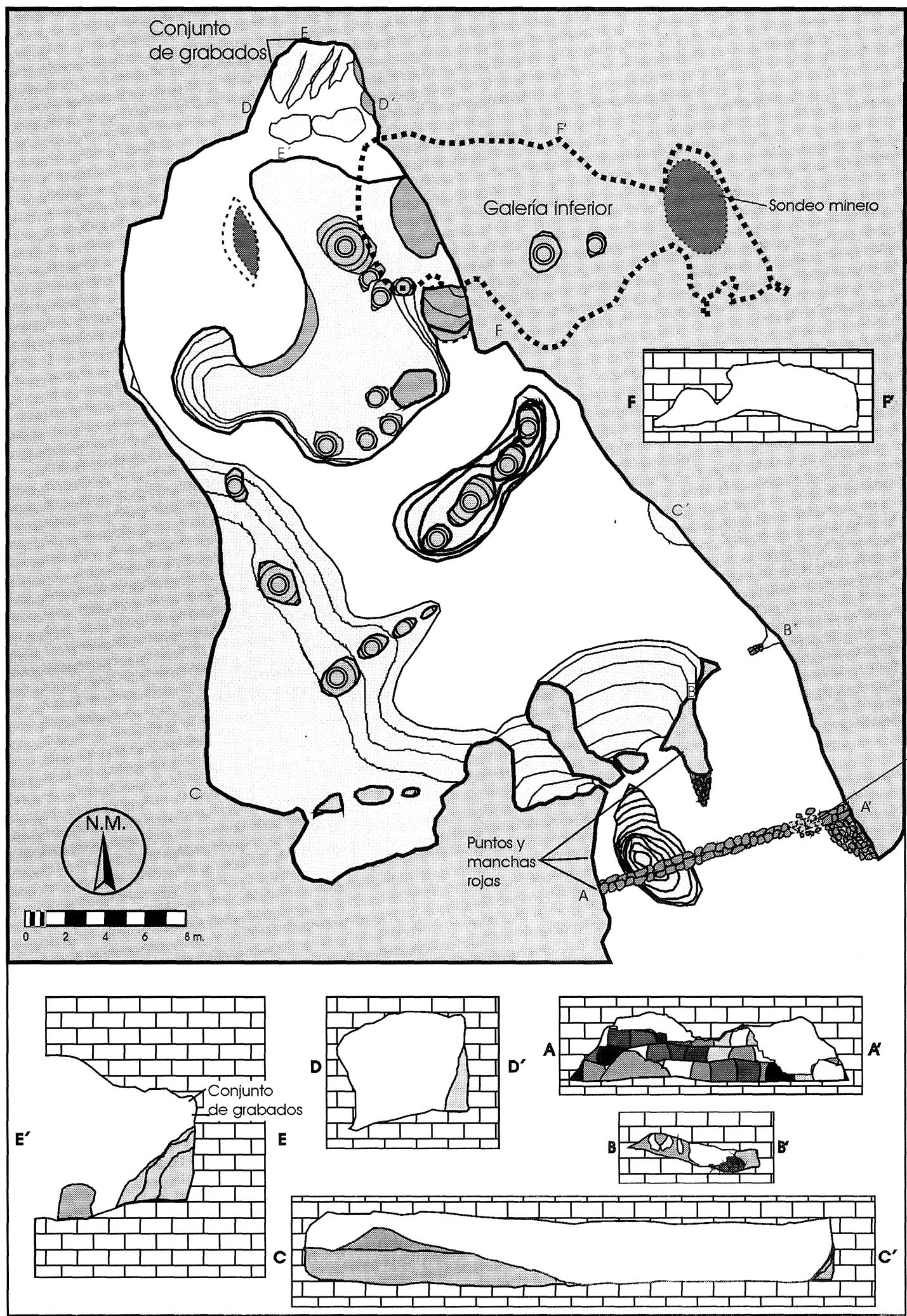

Fig. 2. Topografía de Los Moros de San Vitores con situación de sus manifestaciones rupestres. 
nes litogenéticas, con abundantes coladas, estalactitas, columnas y estalagmitas diseminadas por toda la sala.

Desde la parte derecha de la sala, y a través de una potente colada estalagmítica, se accede a una pequeña galería inferior. Esta galería se ubica unos $6 \mathrm{~m}$ por debajo de la sala principal y tiene un desarrollo lineal de unos $20 \mathrm{~m}$ de longitud, por $10 \mathrm{~m}$ de anchura. Presenta un importante relleno de arcillas de decalcificación, ricas en elementos ferruginosos, en el cual se observa una calicata de gran tamaño que parece corresponderse con un sondeo minero.

\section{LAS INVESTIGACIONES EN LA CUEVA}

La cueva de Los Moros fue descubierta por Luis Salguero, a principios del siglo XX, si bien los primeros trabajos arqueológicos en la misma se deben a Orestes Cendrero (1915), quien da a conocer el hallazgo de industrias paleolíticas procedentes de un nivel ubicado a unos 40 ó $50 \mathrm{~cm}$ de profundidad, industrias que atribuyó al Musteriense.

En la década de los veinte, el padre Jesús Carballo practica excavaciones en la parte izquierda del vestíbulo, según recordaban aún algunos lugareños. Aunque estos trabajos nunca fueron publicados de manera específica, el autor menciona el yacimiento en varias ocasiones (Carballo, 1922 (1), 1924), citando la existencia de evidencias del Musteriense y elAuriñaciense -con azagayas ahorquilladas de base hendida-.

Mayor precisión existe acerca de las investigaciones realizadas en similares fechas por Julio Fernández Montes (1936) (2). De los materiales por él recuperados en la cavidad que nos ocupa se conserva una detallada descripción en un catálogo manuscrito conservado en el Museo Regional de Arqueología y Prehistoria de Santander; entre estas evidencias destacan abundantes restos humanos y fragmentos cerámicos, por lo que todo parece indicar que existía un depósito funerario de la Prehistoria Reciente en algún punto de la cueva.

(1) J. Carballo (1922): El Paleolítico en la Costa Cantábrica. Memoria presentada para aspirar al grado de Doctor de la Facultad de Ciencias (Sección de Naturales). Universidad Central, Facultad de Ciencias. Madrid (mecanografiado inédito, conservado en el Museo Regional de Prehistoria y Arqueología de Santander).

(2) Fernández Montes, J. (1936): Catálogo de Prehistoria. Colección Francisco Fernández Montes (manuscrito inédito conservado en el Museo Regional de Prehistoria y Arqueología de Santander).

T. P., 58, n. ${ }^{\circ} 2,2001$
Aunque no está documentado, es probable que el equipo de Camineros de la Diputación que dirigía el ingeniero L. García Lorenzo, pudiera haber realizado alguna intervención en la cueva durante los años 50-60, ya que esta zona fue intensamente prospectada por ellos y se conocen intervenciones suyas en cavidades próximas.

En los años 60, la Sección de Espeleología del Seminario Sautuola (S.E.S.S.) realiza un primer levantamiento topográfico de la cavidad, durante el cual se localizan restos de una vasija periforme de cuello cilíndrico y asa de cinta, característica de momentos evolucionados de la Edad Media, así como un cráneo humano (Muñoz et alii, 1988). Esta intervención se completó con la realización de algunos pequeños sondeos, algunos de los cuales aún son visibles en el vestíbulo y en la sala interior de la cueva, si bien no se tienen noticias sobre los resultados de los mismos.

A principios de los años 80, el Colectivo para la Ampliación de Estudios de Arqueología Prehistórica (C.A.E.A.P.) hace una detallada descripción del yacimiento del vestíbulo, en la cual se señala el hecho de que, en ese momento, ya no se conservaban restos de los cortes de las intervenciones antiguas. No obstante, este equipo descubre restos de un conchero Mesolítico en el cono de derrubios que desde el vestíbulo cae hacia el interior, y del que se conserva una muestra en el Museo de Santander. También se documenta en ese momento la existencia, en la pared izquierda del vestíbulo, de dos gruesos puntos rojos, aunque su atribución al paleolítico se deja en suspenso (Muñozet alii, 1988).

\section{CONTEXTO ARQUEOLÓGICO INMEDIATO DE LA CUEVA DE LOS MOROS}

En las dos vertientes de Peña Cabarga son abundantes las grutas naturales, muchas de ellas con evidencias arqueológicas (FernándezAcebo, 1983) (3).Así, se conocen una serie de yacimientos en la zona oeste del macizo con depósitos del Paleolítico Superior, como son las cuevas de Morín (González Echegaray y Freeman, 1971), Castañera III y $I V$ (Muñoz et alii, 1988) y La Llosa (Muñoz et alii, e.p.).

(3) V. Fernández Acebo (1983): Peña Cabarga (mecanografiado inédito depositado en los archivos del C.A.E.A.P. Santander). 
Al noroeste, en la margen occidental de la Bahía de Santander, se encuentra el valle de Camargo, con importantes estaciones del Paleolítico Superior, entre las que destacan las cuevas de El Pendo (González Echegaray et alii, 1980; Montes et alii, 1998), El Juyo (Janssens et alii, 1958), El Mazo (Azcuénaga Vierna, 1976) o El Ruso (Muñoz Fernández, 1991), todas ellas con Magdaleniense (excepto el Ruso) y Solutrense (excepto el Juyo), además de niveles del Paleolítico Superior Inicial.

En el propio curso bajo del Miera se encuentran otras cavidades con niveles del Paleolítico Superior, como las cuevas de La Iglesia Iy II (Navajeda) -con Paleolítico Superior Inicial- (Muñoz y Serna Gancedo, 1995), la cueva de la Fuente del Francés (Hoznayo), con Solutrense y Magdaleniense (Obermaier, 1925), y las cuevas del complejo kárstico de La Garma (V.V.A.A., 1999).

Hay que reseñar la presencia, entre todos estos yacimientos, de cinco conjuntos de arte rupestre
Paleolítico, localizados en las cavidades de La Llosa, El Pendo, Alto del Peñajorao, El Juyo y La Garma (Fig. 3).

\section{EL YACIMIENTO ARQUEOLÓGICO DE LOS MOROS DE SAN VITORES}

A pesar de haberse publicado un gran número de citas sobre la cueva, la ausencia de intervenciones arqueológicas recientes y la pérdida, tanto de los materiales arqueológicos, como de las referencias sobre los mismos, ha producido que el conocimiento sobre el yacimiento siga siendo deficitario en la actualidad.

Se ha documentado la presencia de depósito arqueológico en la parte izquierda del vestíbulo y en el cono que, desde este punto, cae hacia el interior de la cueva. Del interior de la cueva tan sólo se tiene noticia de la aparición de un cráneo humano y una vasija medieval.

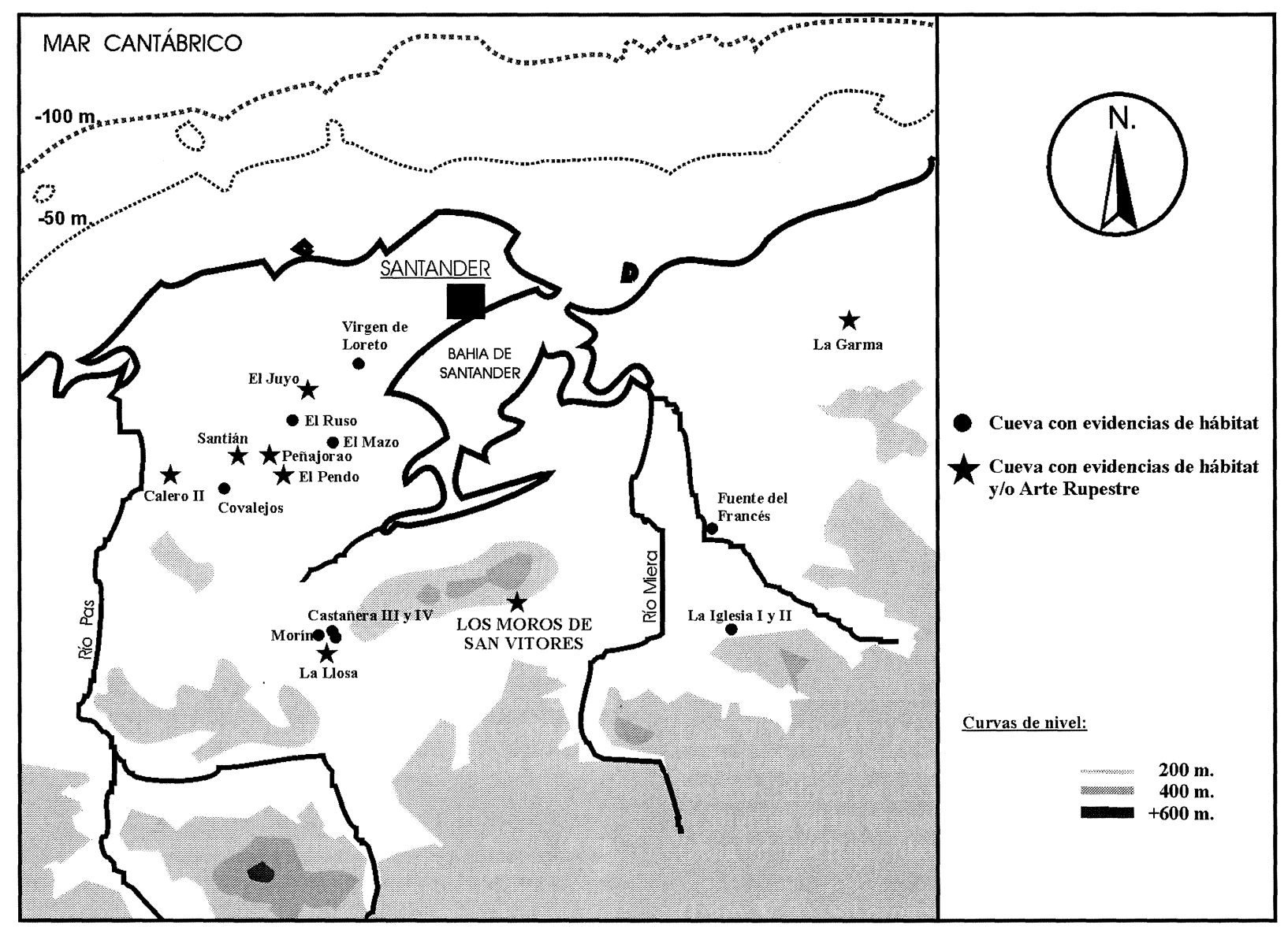

Fig. 3. Localización de la cueva de Los Moros de San Vitores y de los principales yacimientos del paleolítico Superior en el entorno de la Bahía de Santander. 


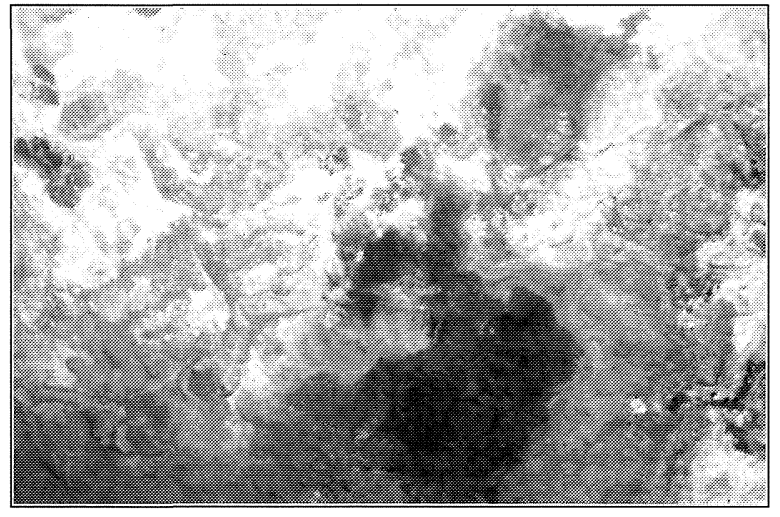

Lám. I. Vestíbulo de la cavidad. Disco rojo (número 2).

Se han citado industrias musterienses y auriñacienses (en la actualidad en paradero desconocido), de las que únicamente se conocen algunos ejemplares líticos dibujados por J. Cabré (una lámina con retoques profundos en ambos bordes, tres raederas y una punta musteriense) y atribuidos al Musteriense (Cendrero, 1915). Es de gran interés la referencia de Carballo (1924) a la existencia de azagayas de base hendida en uno de los niveles de la cavidad, piezas muy características del complejo industrial Auriñaciense.

Mayores precisiones hay sobre el conchero del cono de derrubios, en el que el CAEAP recogió una muestra en superficie que contenía 29 Patella $(13$ vulgata, 14intermedia y 2 ulysiponensis), 35 Mytilus edulis, 3 Venerupis decussata, 3 Solem marginatus, 3 Ostrea edulis, 5 Cepaea nemoralis, esquirlas $y$ huesos fragmentados y un fragmento de posible fémur humano. La composición malacológica del conchero es característica del Mesolítico post-Aziliense, dada la aparición de especies típicamente holocénicas y donde, además de especies de roca, aparecen especies de estuario (tanto de roca como de basa).

También esta documentada la presencia de inhumaciones de la Prehistoria Reciente, probablemente del Calcolítico o la Edad del Bronce, con algunas cerámicas y varias piezas óseas poco características, en donde tan sólo destaca una pieza apuntada con oquedad en el extremo que ha sido identificado como un posible instrumento de estampillar.

Por último, y procedente del interior, hay una jarra con asa de cinta realizada a torno con cocción oxidante. La pieza presenta perfil periforme, con el fondo plano, cuello alto y ligeramente abierto, y labio moldurado hacia el interior. Este tipo de cerámica aparece con cierta frecuencia en cuevas de
Cantabria, en ocasiones asociada a jarras de boca cuadrada, por lo que podemos establecer para ellas una cronología Pleno-medieval.

\section{DESCRIPCIÓN DE LAS MANIFESTACIONES RUPESTRES}

Las muestras de arte rupestre paleolítico documentadas se concentran en dos ubicaciones muy concretas de la cavidad: la parte izquierda del vestíbulo (puntos rojos descubiertos por el CAEAP), $y$ en un recodo situado al fondo de la gran sala interior (conjunto de grabados) (Fig. 2). Al margen, debemos reseñar algunas marcas negras aisladas que, por sus caracteres y localización, no parecen tener relación con las manifestaciones paleolíticas.

En el vestíbulo se han catalogado tres pinturas rojas:

1. Pequeña mancha roja, bastante perdida, situada en el techo junto a la pared izquierda, a 2,5 $\mathrm{m}$ de altura con referencia al suelo del vestíbulo (aunque se accede a él desde un gran bloque calizo). Actualmente es poco visible al quedar semioculto por el muro de cierre.

2. Muy cerca de la anterior, a tan sólo un metro de la boca, y en el techo situado junto a la pared izquierda (a 0,5 $\mathrm{m}$ de altura sobre el suelo) se encuentra un grueso disco rojo, de color intenso y bien conservado. Tiene un grosor de unos $5 \mathrm{~cm}$ de diámetro (Lám. I).

3. En la pared derecha del saliente rocoso del techo que divide el vestíbulo en dos se aprecian restos de una mancha roja bastante perdida. Se ubica a $1 \mathrm{~m}$ del suelo.

Estas pinturas son muy discretas y todas ellas han sido realizadas en zonas bajas del techo, en la parte izquierda de este espacio. Para su ejecución se ha utilizado un ocre de color rojo intenso.

El conjunto interior se presenta sobre un friso lateral ubicado a unos $45 \mathrm{~m}$ al interior, situándose a más de $4 \mathrm{~m}$. de altura, aunque su acceso es cómodo al ser posible acceder al mismo por una serie de crestas rocosas -restos de una diaclasa-, que actúan a modo de escalera natural. En él aparecen grabados incisos (a excepción del grueso punto rojo) de una gran uniformidad técnica, y posiblemente, cronocultural.

Las figuras aparecen dispuestas a lo largo de un panel ubicado entre los 4,5 y $5 \mathrm{~m}$ de altura respecto al suelo actual de la cueva. Se trata de un pequeño friso de roca caliza compacta recubierta por una fina

T. P., 58, n. ${ }^{\circ} 2,2001$ 


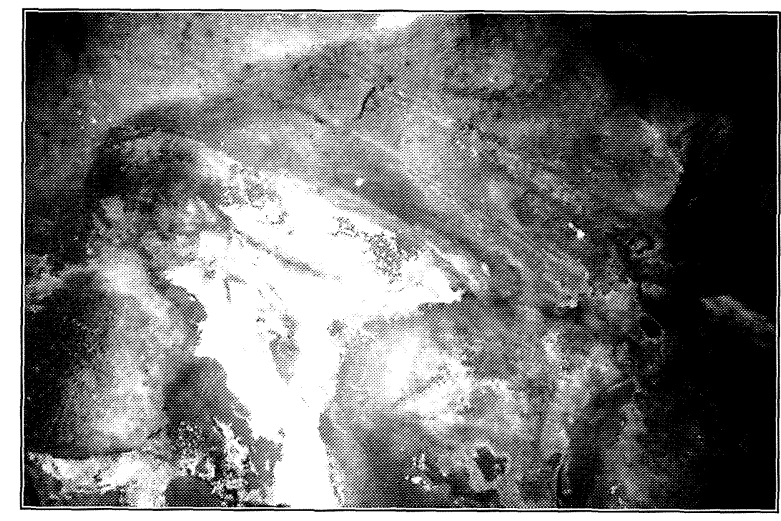

Lám. II. Panel de grabados. Aspecto general.

capa de calcita amarillenta, parcialmente desmantelada por fenómenos de desconchado y pérdida de materiales cubrientes de la roca encajante, y en la que la coloración rojiza, debida a los componentes férricos disueltos en las arcillas de la cavidad, se presenta en forma de película arcillosa de color ocre-rojizo claro. Algunas coladas y precipitaciones calcíticas recientes, generalmente de muy escaso tamaño y desarrollo, completan la superficie del friso-soporte (Lám. II).

Las figuras han sido realizadas -a buril-, mediante trazos finos y únicos, presentando un grabado nítido especialmente destacado en las zonas recubiertas por la película de arcilla de tono ocre-rojizo, por el contraste de coloración entre la superficie y el fondo del surco que, alcanzando la roca encajante, se presenta con un color mucho más claro (casi blanquecino).

En este friso (Fig. 4) se han representado dos bisontes mirando hacia la derecha (hacia el interior de la cueva) en los extremos laterales del friso. Entre ambos bisontes aparecen dos cuadrúpedos: el primero mira a la izquierda (es decir, hacia la entrada de la cueva), y aunque se encuentra deficientemente conservado - por lo que no se ha podido clasificar con mayor precisión-, todo apunta a que se trata de los cuartos traseros de un cérvido. Inmediatamente a la derecha de éste último, se localiza la figura del équido grabado en vertical, que mira ha-

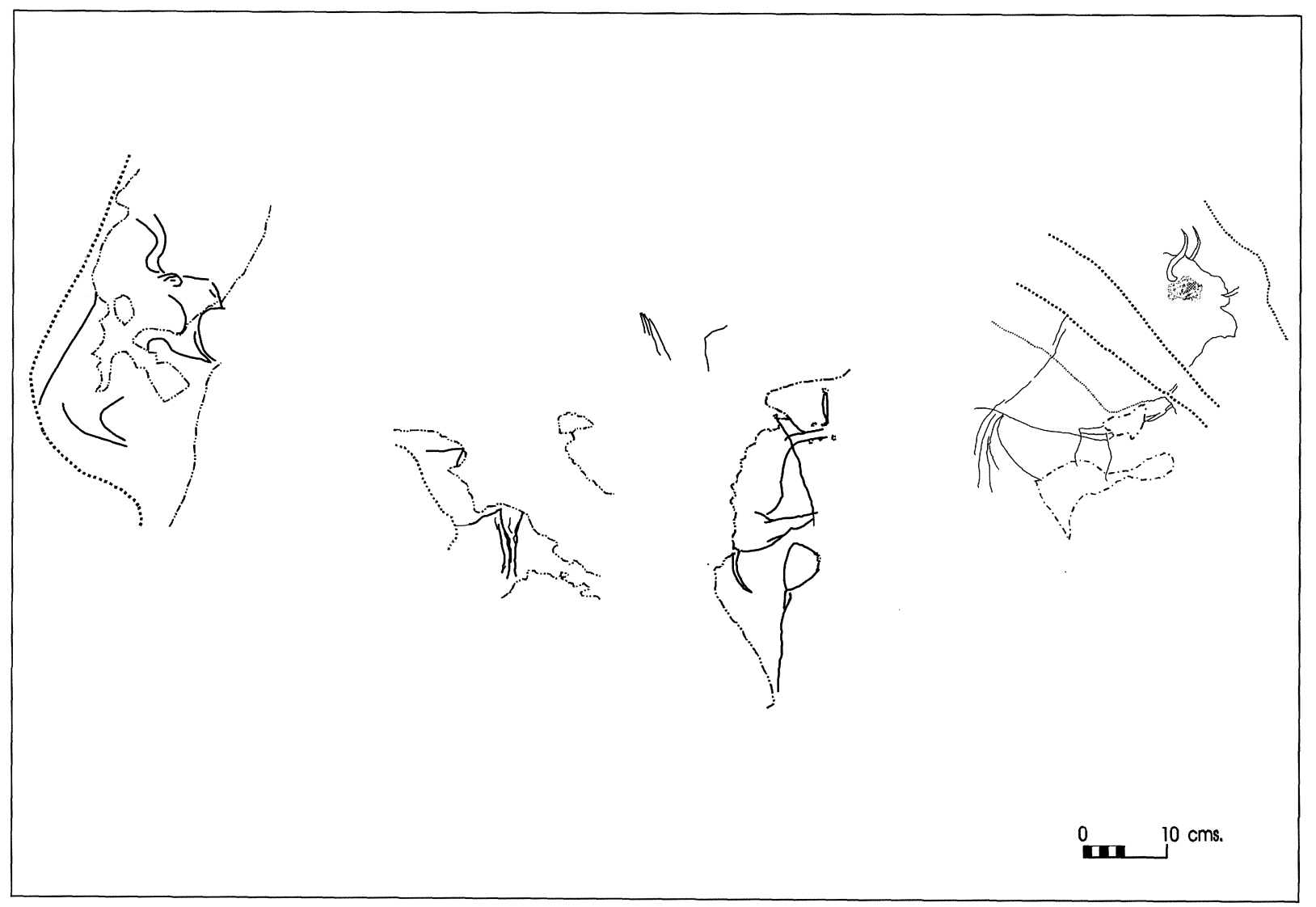

Fig. 4. Panel de los grabados. Disposición del registro parietal. 


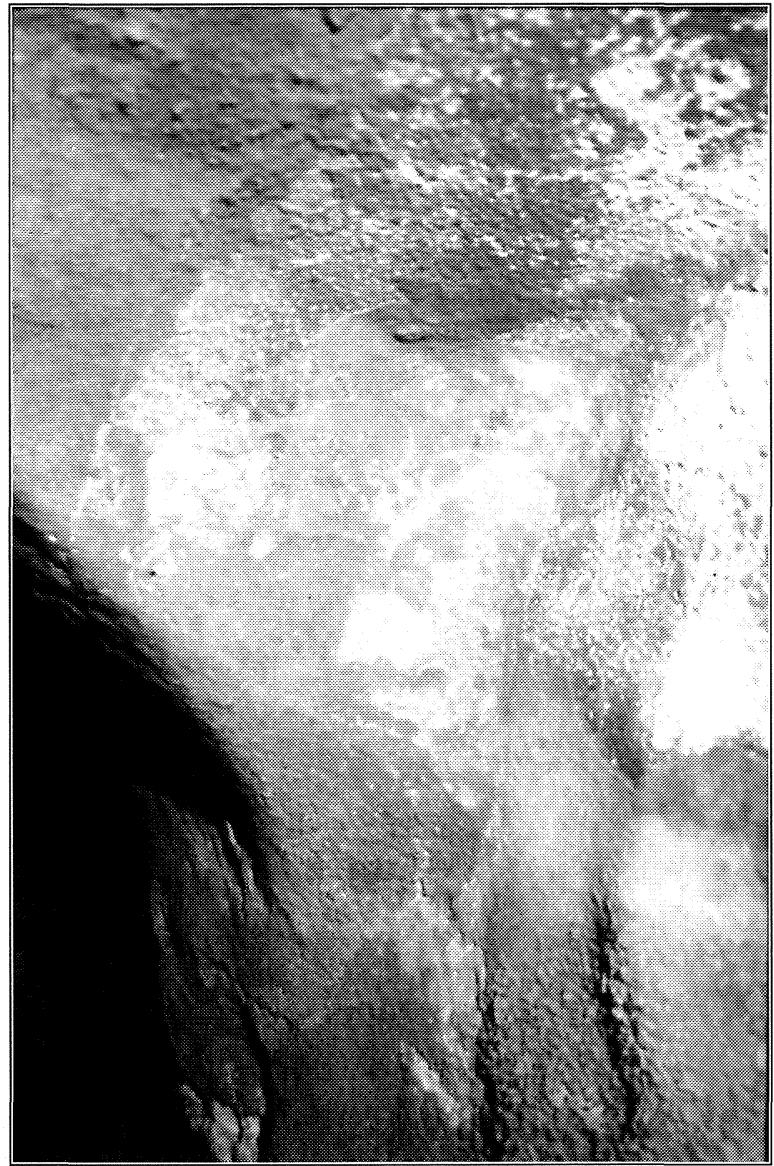

Lám. III. Panel de grabados. Figura de bisonte (?) (número 1$)$.

cia la derecha y que en la actualidad se presenta como una figura acéfala, debido a la pérdida de la capa superficial de calcita sobre la que fue grabado en las zonas en donde se localizarían la cabeza y buena parte de la línea cérvico-dorsal. Por encima de estas dos últimas figuras hay algunas líneas aisladas, quizás pruebas de buril. Por último tendríamos un signo cerrado prolongado por una línea vertical, inmediatamente a la derecha del caballo, si bien en un plano inferior.

Se han reconocido, por tanto, las siguientes figuras (de izquierda a derecha):

1. Figura incompleta y poco visible realizada con trazo fino y único. Se trata de una representación asignada, con muchas dudas, a la figura de un bisonte, $y$ en donde se han distinguido los cuernos -sinuosos y en perspectiva correcta-, y la cara (incluida la barba). Del resto de la figura se aprecia únicamente una posible grupa, la pata trasera y la parte posterior del vientre. El trazo es nítido, espe-

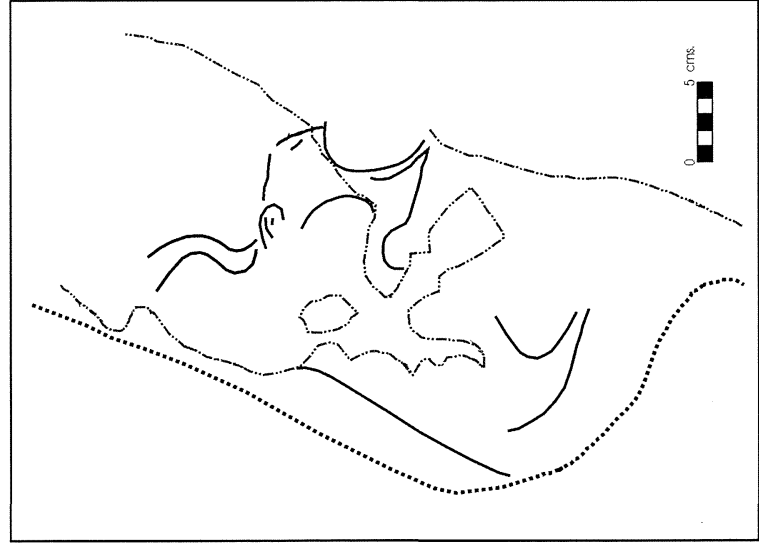

Fig. 5. Representación de bisonte (?), número 1.

cialmente en los cuernos y en la parte baja de la cabeza (Lám. III). La figura aparece dispuesta en vertical, con la cabeza hacia arriba y mirando hacia la derecha. Mide unos $35 \mathrm{~cm}$ (Fig. 5).

2.'Figura incompleta realizada en grabado fino, inciso y único, más nítido y marcado en las extremidades (Lám. IV). Representa, sin duda alguna, los cuartos traseros de un cuadrúpedo. La figura parece que pudo haber estado representada de forma completa, si bien la pérdida parcial de la película de calcita que recubre la pared en este punto, ha podido hacer desaparecer los detalles anatómicos que faltan. Únicamente se conserva parte del lomo, la parte posterior del vientre y las patas traseras, paralelas y en perspectiva correcta, de lo que fue la representación. Se trata de una figura bastante maciza, con el vientre prominente. La patas traseras miden $10 \mathrm{~cm}$ y la anchura del animal desde la línea del dorso hasta el vientre es de $12 \mathrm{~cm}$. Si bien resulta problemática una asignación taxonómica, ante lo limitado de la manifestación, consideramos que la misma pudiera corresponderse con los cuartos traseros de un cérvido (Fig. 6). Sobre esta última cuestión volveremos más adelante.

3. Figura incompleta de un cuadrúpedo, dispuesto en vertical. La figura tiene un trazo único y nítido, sin correcciones. La película de calcita de la pared ha desaparecido en la parte izquierda de la manifestación, haciendo desaparecer la línea cérvico-dorsal y la cabeza del animal que, muy posiblemente, también estaban representadas (Lám. V). Presenta un rabo ligeramente levantado y apuntado, grupa, pata trasera, vientre poco sinuoso, pata delantera indicada con dos trazos y parte posterior del cuello. Pese a las limitaciones para una identifica- 


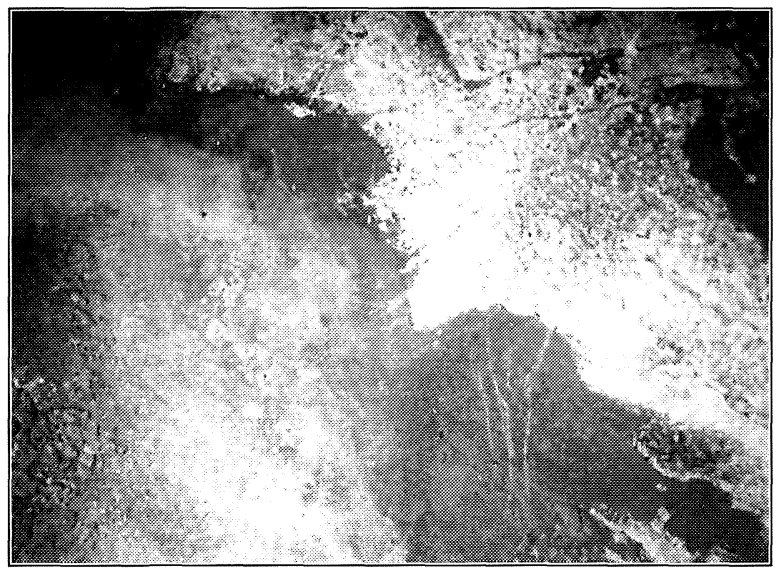

Lám. IV. Panel de grabados. Cuartos traseros de cuadrúpedo (¿cérvido?, número 2).

ción taxonómica, todo apunta hacia la representación de un équido, como interpretación más plausible (Fig. 7 a).

La manifestación está atravesada por una línea oblicua grabada (¿azagaya?) de $16 \mathrm{~cm}$ que recorre desde el cuello hasta la parte posterior de la pata delantera. La parte conservada mide $23 \mathrm{~cm}$ de anchura (desde el extremo de la cola al cuello) y $9 \mathrm{~cm}$ de altura (desde la parte alta de la grupa hasta la base de la pata trasera).

4. Inmediatamente por encima de la figura anterior, son visible dos líneas finas, oblicuas, muy próximas entre sí, de unos $5 \mathrm{~cm}$ de longitud.

5. Por encima de las dos figuras anteriores, se documenta un grupo de líneas sueltas, cuatro oblicuas y casi paralelas a la izquierda, y una línea en ángulo a la derecha (Fig. 7 b). Estos trazos miden 7,4 y $7 \mathrm{~cm}$, respectivamente.

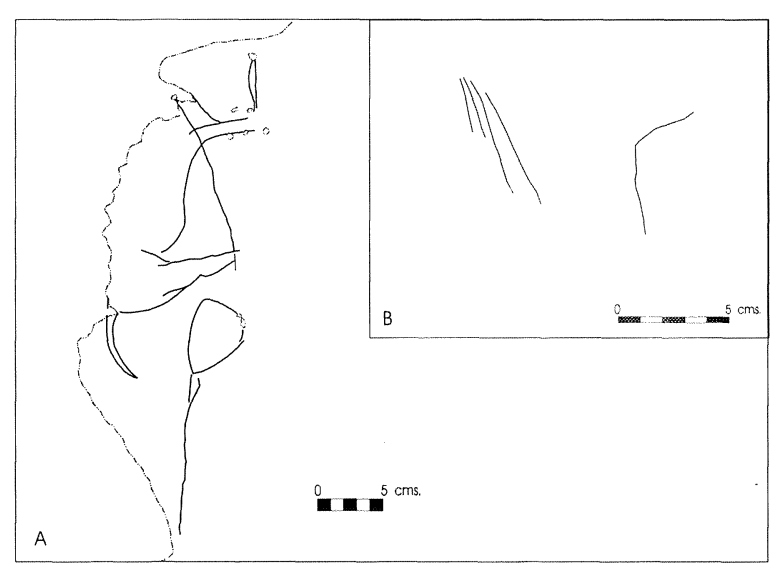

Fig. 7.A) Representación de équido en vertical, número 3, y posible signo, número 6 . B) Trazos sueltos, número 5 .

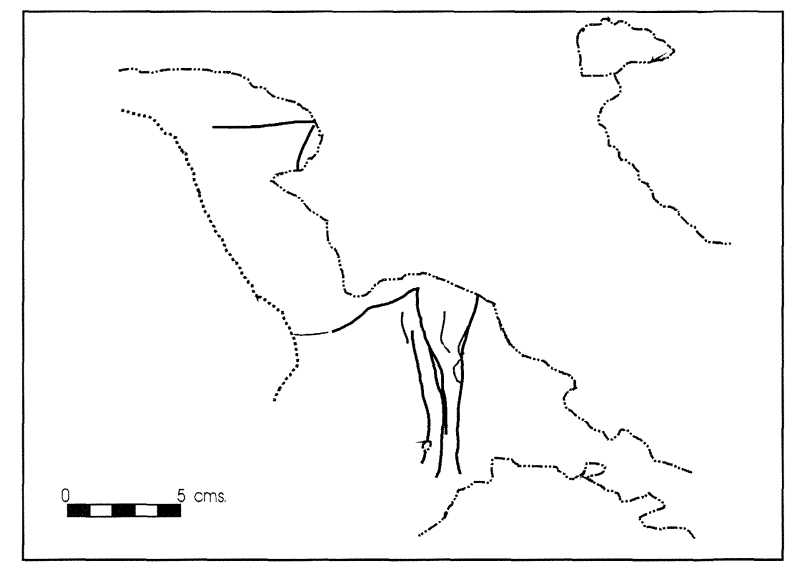

Fig. 6. Cuartos traseros de cuadrúpedo (¿cérvido?), número 2 .

6. Por debajo de las patas traseras de la figura 3 hay una manifestación no figurativa compuesta de un triángulo, formado por tres líneas ligeramente arqueadas, y una línea vertical que se prolonga desde el ángulo inferior del triángulo. En conjunto, la figura mide $15 \mathrm{~cm}$ de altura. Las líneas que forman el triángulo miden entre 3 y $5 \mathrm{~cm}$, mientras que la línea inferior, a modo de prolongación, mide $11 \mathrm{~cm}$. Esta manifestación ha sido identificada, no sin ciertas reservas, como un signo (Fig. 7 a).

7. Figura de bisonte completo grabado en trazo simple, único y muy fino, sobre todo en la zona de la cabeza (Lam.VI). La figura se encuentra entre 4,3 y 4,9 m de altura sobre el suelo de la sala, y está dispuesta diagonalmente con la cabeza hacia arriba, mirando hacia la derecha. Presenta una gran cabeza, con barba puntiaguda, el perfil de la cara (en la que destaca la lengua lanceolada) y dos cuernos sinuosos y paralelos (con dos trazos cada uno que parten del interior del contorno).

A la altura del ojo se dispone un grueso disco rojo pintado que, probablemente, sirve para indicarlo. El arranque de la giba es muy poco visible al estar realizado con un trazo muy fino, y la giba propiamente dicha, no es apreciable. La grupa, por el contrario, es fácilmente distinguible. La cola es corta $(8 \mathrm{~cm})$, ligeramente arqueada y ha sido realizada mediante varios trazos paralelos. La pata posterior está parcialmente recubierta por una concreción, por lo que tan sólo es visible su arranque. El vientre está realizado mediante una línea suave y fina, apenas perceptible, al igual que la pata delantera y el cuello.

Al presentarse en distintos planos de la roca, no es posible ofrecer unas medidas totales de la figu- 


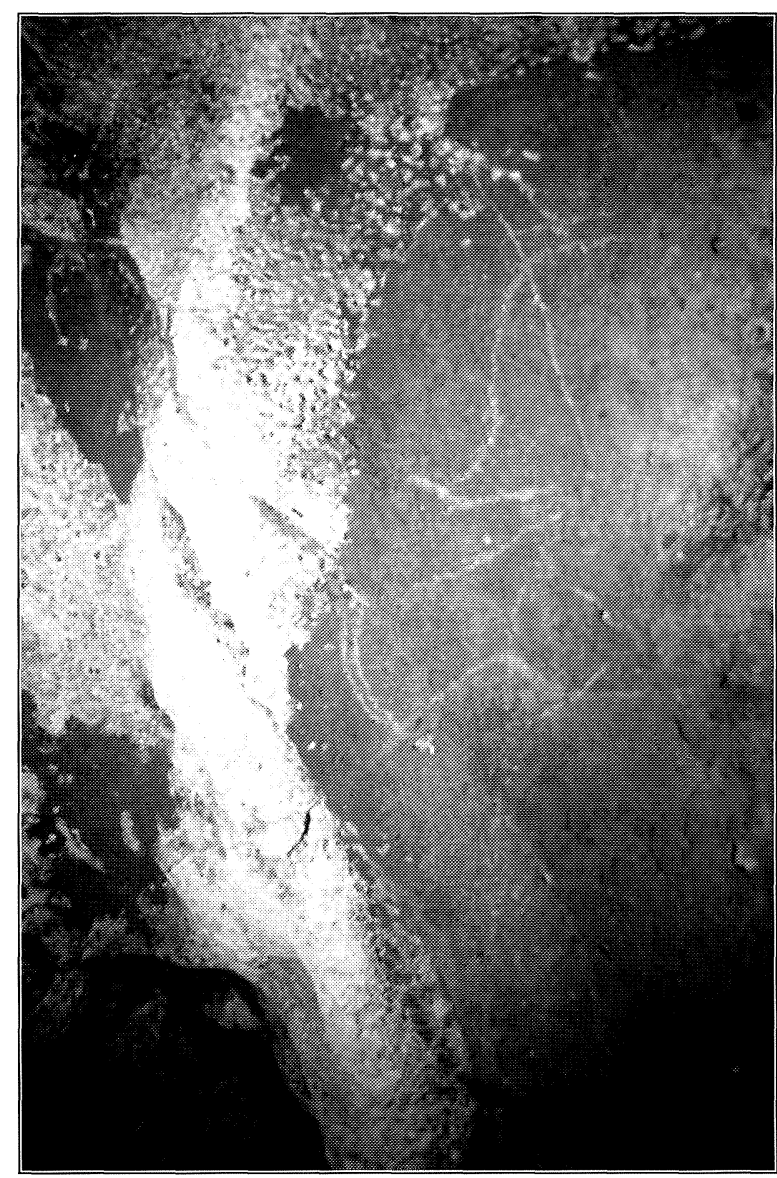

Lam. V. Panel de grabados. Representación de équido en vertical (número 3 ) y posible signo (número 6).

ra, por lo que debemos despiezar la misma a la hora de ofrecer sus dimensiones. La parte trasera del animal mide $19 \mathrm{~cm}$, contando desde el final de la giba hasta la pata trasera. La altura del tren trasero del animal es igualmente de $19 \mathrm{~cm}$. Desde la zona inguinal hasta el arranque de la pata delantera hay $14 \mathrm{~cm}$. La cabeza, desde el extremo del cuerno, hasta la punta de la barba, mide $22 \mathrm{~cm}$ (Fig. 8).

Además de estas figuras, de clara asignación paleolítica, se han localizado en dos lugares diferentes de la cavidad dos pequeños conjuntos de marcas negras, compuestas por líneas cortas realizadas con carbón vegetal. El primero se localiza en un friso muy aparente próximo a la bajada a la galería inferior, a 1,6 m del suelo. El segundo, en una oquedad abierta de forma natural, y ubicada a ras de suelo, en la pared izquierda de la sala principal. No obstante, estas manifestaciones no parecen estar relacionadas con el conjunto parietal paleolítico, y posiblemente estén en conexión con las manifesta-

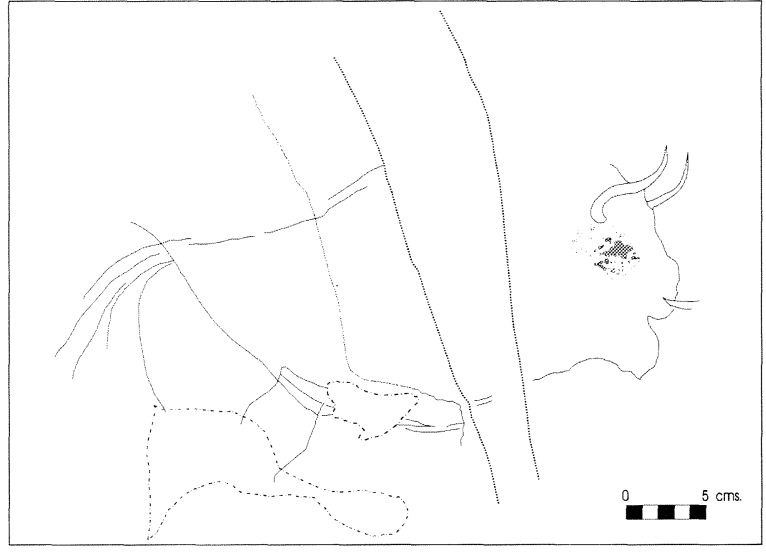

Fig. 8. Representación completa de bisonte con mancha roja a la altura del ojo, número 7 .

ciones de la Prehistoria Reciente, o más seguramente, con las evidencias medievales detectadas en la gruta.

En cuanto a la temática y a la realización formal de las unidades gráficas documentadas podemos exponer lo siguiente:

Las representaciones del vestíbulo son muy simples; en ellas se distingue un grueso disco rojo y unas reducidas manchas, quizás restos de otros discos mal conservados, igualmente rojos. Las representaciones de discos son numerosas en los conjuntos rupestres paleolíticos cantábricos, tanto de manera aislada, como formando agrupaciones. Suelen aparecer en distintos lugares topográficos, desde los vestíbulos, hasta en el fondo de las cavidades.

Las asociaciones de pequeños conjuntos de pinturas rojas no figurativas a la entrada de una cueva con representaciones más complejas ubicadas en el

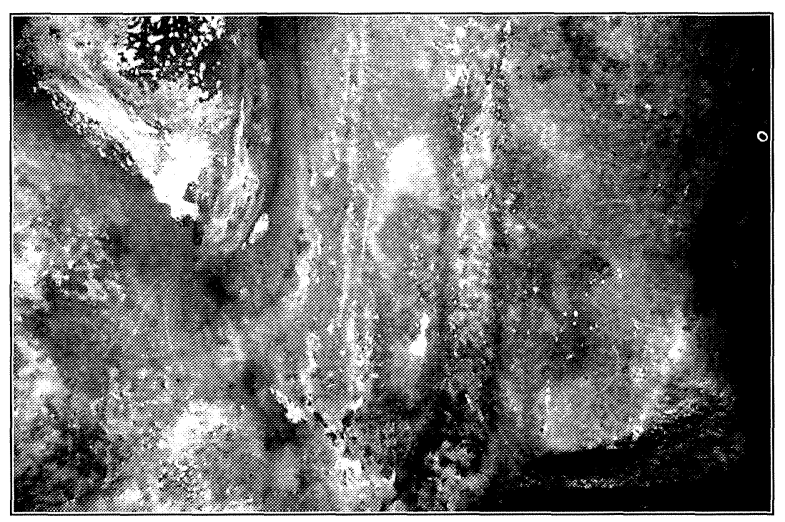

Lam. VI. Panel de grabados. Representación completa de bisonte con mancha roja a la altura del ojo (número 7). 
interior, como el caso que nos ocupa, son relativamente frecuentes en la Región Cantábrica, si bien en conjuntos propios de la fase Graveto-Solutrense, como en el caso de las Cuevas delArco A y Arco B, en Pondra (Muñoz et alii, 1991), o la Cueva de El Pendo (comunicación personal de R. Montes).

En cuanto a las figuras del interior, el animal más representado es el bisonte, con dos ejemplares. Ambos presentan rasgos muy característicos y similares, especialmente en la cabeza, con cuernos muy sinuosos y una línea de la cara quebrada con indicación de barba puntiaguda. Es interesante reseñar que el bisonte situado en el extremo derecho de la composición tiene detallada la lengua. Como es sabido, la representación de la lengua aparece circunscrita a figuras de bisontes de cuevas pirenaicas -Trois Frères, Marsoulas, Le Portel-y del Cantábrico - Hornos de la Peña, Altamira, Pasiega, Covaciella, Llonín y San Román de Candamo- (Fortea et alii, 1995). El resto del cuerpo de esta figura se presenta en trazo más fino y sumario, e incluso más bien desmañado. La giba, a diferencia de la mayor parte de las representaciones de este animal en el arte franco-cantábrico, es poco prominente y apenas si está bien reseñada.

En el centro del panel se halla la figura de un équido (caballo) en vertical, trazado mediante un grabado firme, nítido y más grueso, con una línea oblicua que parte del pecho y que se ha interpretado como una posible azagaya clavada (como ocurre con el caballo pintado en negro en la cueva de Sotarriza). La figura, aunque de correctas proporciones, es bastante simple en su elaboración.

El animal restante no ha podido ser bien identificado, debido a la mala conservación de la pared, pues conserva tan sólo los cuartos traseros. Se trata, en todo caso, de un cuadrúpedo con proporciones idóneas para ser asignado a la figura de un cérvido, ya que por las características de la representación puede descartarse que se trate de otro bisonte. Tan sólo la posibilidad de que se tratase de un segundo caballo pone en duda esta atribución taxonómica.

Al margen de los animales, aparece un signo triangular cerrado que se prolonga mediante un trazo vertical. Se sitúa junto al caballo, en clara asociación con él. Parece tratarse de un signo acoplado, que estaría formado por la combinación de un signo cerrado femenino y uno abierto masculino. Estos signos son relativamente abundantes en los conjuntos paleolíticos, tanto en el arte parietal, como en el mueble, y dada la simplicidad del motivo resulta difícil establecer paralelos exactos. Algunos de los más ajustados se encuentran en las cuevas francesas de Oulen y en Saint Marcel (Leroi-Gourhan, 1963), aunque más bien podríamos hablar de convergencias formales, y no necesariamente de signos con el mismo significado.

Otros grabados lineales situados en la parte alta del panel, y que no forman motivos aparentes, pueden ser identificados como simples pruebas de buril.

En cuanto a las técnicas empleadas, podemos exponer lo siguiente:

- La pintura roja de las manifestaciones exteriores y del punto localizado a la altura del bisonte $\mathrm{n}^{\circ}$ 7 , parece haber sido aplicada de manera directa y no mediante la técnica del soplado. Esta impresión se debe al hecho de que en todos los casos los perfiles son bastante nítidos y en ningún caso presentan el aspecto difuminado propio de las manifestaciones realizadas con pintura soplada.

- Los grabados, por su parte, han sido realizados a base de trazos simples y únicos que, en casos puntuales, aparecen repetidos -tal y como ocurre en la cola del bisonte $\mathrm{n}^{\circ} 7$-. Los grabados presentan un ligero tono blanquecino, al haber atravesado la capa de calcita ocre-amarilla que recubría totalmente la roca, y alcanzar la roca encajante. En cuanto al grosor, hay diferencias que tal vez estén relacionadas con el tipo de soporte. En este sentido, destaca las diferencias en el trazo del bisonte $n^{\circ} 7$, en el que la parte delantera está realizada con un trazo ancho y nítido, mientras que la trasera presenta un trazo mucho más fino.

El campo manual de las representaciones es prácticamente similar. Las figuras han podido ser realizadas con el brazo ligeramente extendido, y de una forma cómoda desde la plataforma pétrea ubicada al pie del panel (Fig. 2, sección E-E').

Probablemente todo el conjunto de grabados sea sincrónico ya que, además de ocupar un lugar muy concreto y delimitado de la cueva, presentan una gran similitud estilística y técnica, como veremos a continuación.

Desde un punto de vista crono-estilístico, y en una visión muy sintética, podemos exponer:

1. Las pinturas rojas de la entrada son de compleja asignación crono-cultural, dados los argumentos dirimidos con anterioridad y su dudosa relación con el conjunto del interior, pese a la concurrencia en ambos conjuntos de discos de color rojo. 
Como es sabido, los discos tienen escaso valor cronológico (al aparecer a lo largo de todo el Paleolítico), si bien parecen ser más frecuentes en los conjuntos presuntamente más antiguos, donde a veces constituyen el motivo más frecuente, como es el caso de las cercanas cuevas de Cudón (Muñoz et alii, 1991) y Calero II (Muñoz y Morlote, e.p.).

2. En cuanto a las representaciones grabadas podemos decir que encajan perfectamente en los caracteres que definen el estilo IV de Leroi-Gourhan, con cuadrúpedos completos con perspectiva correcta en los cuernos y líneas cérvico-dorsales poco marcadas y poco sinuosas.

Sin duda alguna, la figura más expresiva desde el punto de vista crono-estilístico es el bisonte de mayor tamaño (n. ${ }^{\circ}$ ), especialmente en los detalles anatómicos de la cabeza, como los cuernos, la barba, y sobre todo la representación de la lengua, motivo que se restringe (según la sistematización deA. Leroi-Gourhan, 1963) a conjuntos de estilo IVAntiguo, en sus momentos iniciales. No obstante, algunos de los conjuntos que ejemplifican este estilo se han fechado en el Magdaleniense Medio (Hornos de la Peña, Covaciella, Llonín, Altamira, etc...), destacando en este sentido los de Covaciella y Altamira, algunas de cuyas figuras han podido ser fechadas por carbono 14 (Fortea et alii, 1995), en fechas que abarcan entre el 13500 y el 15000 B.P.

3. Además, hay que indicar que en el conjunto de grabados están representados los dos animales más frecuentes en los conjuntos de estilo IV -propios del Magdaleniense- el caballo y el bisonte. Habitualmente, estas manifestaciones forman el motivo principal de los conjuntos, tanto por ser las representaciones más numerosas, como por el lugar que ocupan dentro de los dispositivos parietales. En este sentido, hay que decir que la cueva de Los Moros posee un dispositivo parietal, aunque muy sumario, bastante característico y similar al de este tipo de yacimientos.

4. Si bien no se conocen otros conjuntos de grabados que se correspondan plenamente al esquema compositivo de Los Moros, cada día son más frecuentes los pequeños conjuntos de grabados de cronología magdaleniense, con una serie de características comunes al que nos ocupa, entre las que podemos destacar:

- El empleo del grabado inciso, generalmente de trazo fino y único.
- El tamaño reducido de las representaciones, generalmente de animales, y en donde son frecuentes las representaciones incompletas.

- La aparición en contextos exentos de otro tipo de manifestaciones parietales.

- La situación topográfica de los grabados en las proximidades de las zonas de hábitat, siendo su acceso sencillo, aunque están en lugares generalmente recogidos.

- La aparición de estos conjuntos en cavidades con ricos depósitos de las últimas fases del Paleolítico Superior.

5. Respecto a otros paralelos formales en la región cantábrica que pudieran ayudar a una fijación cronológica hay que decir que, representaciones de este tipo, únicamente han sido detectadas en la zona central (Cantabria), posiblemente debido al menor desarrollo de la prospección delArte Rupestre $\mathrm{Pa}$ leolítico en las áreas oriental y occidental en donde, no obstante, es presumible que también estén presentes. De hecho, en Cantabria todos son hallazgos recientes.

Las cuevas catalogadas con conjuntos de grabados afines al que nos ocupa son: Cueva Grande de Otañes (González Sáinz et alii, 1994); la cueva de Cobrante, en San Miguel de Aras (García Guinea, 1968); la cueva de Sotarraña o El Patatal, en Matienzo (Balbín et alii, 1986); la del Otero, en Secadura (González Sáinz et alii, 1985); la cueva del Juyo, en Igollo (Muñozet alii, e.p.); la cueva de Sovilla (González Sáinz et alii, 1994); y la cueva del Linar, en La Busta (San Miguel, 1991).

Con estos datos, y dada la imposibilidad de afinar mucho más ante lo limitado del discurso cronológico (actualmente en profunda revisión), debemos situar el conjunto de grabados de la Cueva de Los Moros de San Vitores en un arco cronológico amplio, aunque circunscrito al Magdaleniense, que podemos fijar muy grosso modo entre el $12000 \mathrm{y}$ el 15000 B.P. No obstante, no queremos dejar de exponer nuestra impresión de que, a la vista de los caracteres del conjunto (temática y composición principalmente), nos parece razonable proponer una fecha que podríamos ubicar entorno al 14000 B.P. (Magdaleniense Medio), como hipótesis cronológica más probable.

\section{RECAPITULACIÓN FINAL}

La cueva de Los Moros de San Vitores, a pesar de poseer un yacimiento paleolítico ya documentado

T. P., 58, n. ${ }^{\circ} 2,2001$ 
a principios del siglo $\mathrm{XX}$, todavía es poco conocida.Así, y aunque se han atestiguado la presencia de industrias del Paleolítico Superior, en concreto del Auriñaciense (no siendo descartable la existencia de otros momentos, especialmente del Magdaleniense), el enclave carece aún de un mínimo estudio sistemático y específico que incluya el estudio de su secuencia estratigráfica y su valoración en el contexto regional.

Por nuestra parte, en este trabajo únicamente se ha pretendido el dar a conocer el pequeño conjunto de Arte Rupestre Paleolítico recientemente reconocido en la cavidad, como contribución al conocimiento de este interesante yacimiento, hasta fechas recientes prácticamente olvidado.

Como hemos expuesto, el dispositivo parietal de la cueva es muy limitado, con una serie de pequeñas manifestaciones rojas en el vestíbulo y un pequeño panel de grabados asociados a un disco rojo en el interior.

El conjunto interior, el más significativo y reseñable, presenta cuatro animales, un signo complejo y varias líneas aisladas, además del mencionado disco rojo (posiblemente relacionado, como hemos expuesto, con el ojo del bisonte ubicado en el extremo derecho del friso).

La lista de figuraciones se reduce a dos bisontes (aunque uno de ellos no deje de ser algo dudoso), un caballo y un cuadrúpedo (que provisionalmente hemos identificado como un cérvido). Destaca la figura completa del bisonte ubicado en el extremo derecho, el cual presenta, como detalle más significativo, la representación de la lengua (posiblemente lanceolada), detalle anatómico muy característico de algunos conjuntos del Magdaleniense Medio del Cantábrico y de Pirineos.

A partir de sus caracteres, y de la similitud formal de las representaciones con otras de yacimientos mejor datados, se ha establecido una cronología para el conjunto centrada en las fases centrales del período Magdaleniense.

\section{BIBLIOGRAFÍA}

Azcuénaga Vierna, J. (1976): “Cueva de Camargo". En XL Aniversario del Centro de Estudios Montañeses, III. Santander: 337-349.

Balbín, R. de; González Morales, M.R. y González SÁınZ, C. (1986): "Los grabados y pinturas de las cuevas de Los Emboscados y El Patatal (Matienzo, Cantabria)". Estudio de Arte Paleolítico. Memorias del Centro de Investigación y Museo de Altamira, 15. Madrid: 235-270.
Carballo, J. (1924): Prehistoria universal y especial de España. Impr. de la Viuda de L. del Horno. Madrid.

Cendrero, O. (1915): "Resumen de los bastones perforados de la provincia de Santander. Noticias de dos nuevos yacimientos prehistóricos de la provincia de Santander". Boletín de la Real Sociedad Española de Historia Natural, IV. Madrid: notas números 1 y 2.

Fortea, J.; Rodríguez, V.; Hoyos, M.; Federación AsturiaNA DE Espeleología; Valladas, H. y Torres, T. DE (1995): "Covaciella". Excavaciones arqueológicas en Asturias 1991-94. Consejería de Cultura de Asturias. Oviedo: 258-270.

García Guinea, M.A. (1968): Los grabados de la cueva de la Peña del Cuco en Castro Urdiales y de la cueva de Cobrantes (Valle de Aras). Patronato de las Cuevas Prehistóricas de la Provincia de Santander. Santander.

GonZÁlez Echegaray, J. (1980): El yacimiento de la cueva de "El Pendo" (Excavaciones 1953-1957). Bibliotheca Praehistorica Hispana, XVII. Madrid.

GonzÁlez Echegaray, J. y Freeman, L.G. (1971): Cueva Morín. Excavaciones 1966-1968. Patronato de las Cuevas Prehistóricas de la Provincia de Santander. Santander.

González Sáinz, C.; Montes Barquín, R. y Muñoz FerNÁNDEZ, E. (1994): "La cueva de Sovilla (San Felices de Buelna, Cantabria)". Zephyrus, XLVI: 7-36.

González Sáinz, C.; Muñoz Fernández, E. y San Miguel Llamosas, C. (1985): "Los grabados rupestres paleolíticos de la cueva del Otero (Secadura, Cantabria)". Sautuola, IV. Santander: 155-164.

González, C.; Bohigas, R.; Molinero, J.T.; Muñoz, E.; Fernández, M. y Arozmena, J.F. (1994): "La cueva Grande (Otañes, Cantabria). Arte rupestre y yacimiento arqueológico". Trabajos de Arqueología en Cantabria II, Monografías Arqueológicas de la ACDPS, 5. Santander: 32-72.

JansSEns, P.; GonZÁlez Echegaray, J. y AzPeitia, P. (1958): Memoria de las excavaciones de la Cueva del Juyo (1955-1956). Patronato de las Cuevas Prehistóricas de la Provincia de Santander. Santander.

Leroi-Gourhan, A. (1963): Préhistoire de l'art occidental. Edit. d'Art Lucien Mazenod. París.

Montes, R.; SAnguino, J.; Gómez, A. y G. Luque, C. (1998): "Cueva de El Pendo. Nuevas manifestaciones rupestres paleolíticas". Revista de Arqueología, 201: 10-15.

MuÑOZ FERNÁNDEZ, E. (1991): "Excavaciones arqueológicas en la Cueva del Ruso I. Avance preliminar". Arque nas, 1 (Cartas y Yacimientos). Ed. Impresión. Santander: 61-130.

Muñoz Fernández, E.; Montes Barquín, R. y Morlote Expósito, J. M. (e.p.): “Catálogo de cavidades conArte Rupestre Paleolítico de la Comunidad Autónoma de Cantabria: Nuevas aportaciones". I Congreso Internacional de Arte Rupestre (Vigo, diciembre de 1999).

MuÑoz Fernández, E. y Morlote, J.M. (e.p.): "El conjunto rupestre paleolítico de la cueva del Calero II (Oruña, 
Cantabria). Primeros resultados de su estudio". I Congreso Internacional de Arte Rupestre (Vigo, diciembre de 1999).

Muñoz Fernández, E.; San Miguel Llamosas, C. y C.A.E.A.P. (1988): CartaArqueológica de Cantabria. Edit. Tantín. Santander.

Muñoz Fernández, E.; San Miguel Llamosas, C. y Gómez Arozamena, J. (1991): "Avance al estudio del Arte Rupestre Paleolítico de la Caverna de Cudón". Arquenas, 1 (Arte Rupestre y Mobiliar). Santander: 31-88.

Muñoz Fernández, E. y Serna Gancedo, A. (1995): “Yaci- mientos del Paleolítico Superior poco conocidos". Boletín Cántabro de Espeleología, 11: 101-123.

San Miguel Llamosas, C. (1991): "El conjunto de Arte Rupestre Paleolítico de la Cueva de Linar (Alfoz de Lloredo, Cantabria)".Crónica del XX Congreso Nacional de Arqueología (Santander 1990): 95-103. Zaragoza.

OBermaIer, H. (1925): El Hombre Fósil. Comisión de Investigaciones Prehistóricas y Paleontológicas, 9. Madrid.

VV.AA. (1999): La Garma. Un descenso al pasado. Gobierno de Cantabria y Universidad de Cantabria. Santander. 